\title{
Alternate Scenarios Working Group Summary
}

\section{Gautam Bhattacharyya*}

Saha Institute of Nuclear Physics, l/AF Bidhan Nagar, Kolkata 700 064, India

E-mail: Gautam.Bhattacharyya@saha.ac.in

\section{Debajyoti Choudhury}

Dept. of Physics and Astrophysics, University of Delhi, Delhi 110 007, India

E-mail: debchouephysics.du.ac.in

This is a brief summary of the activities/talks in the Alternate Scenarios Working Group. The following topics were discussed: (i) Gauge-Higgs unification (by S.C. Park), (ii) Little Higgs mechanism (by A. Cohen), (iii) $W_{L}-W_{L}$ scattering (by S.D. Rindani), (iv) Mass, mass-shifts, Higgs (by Y.N. Srivastava), (v) Top quark spin correlations (by K. Smolek), and (vi) Single top production at D0 (by V. Simak).

From Strings to LHC

January 2-10 2007

International Centre Dona Paula, Goa India

${ }^{*}$ Speaker. 


\section{Gauge Higgs unification}

S.C. Park gave a talk on some aspects of Gauge Higgs unification. How does extra dimension offers a protection mechanism to the Higgs mass constitutes the basic idea of Gauge-Higgs Unification (GHU). In this formalism, the Higgs boson arises from the internal components of a higher dimensional gauge field. Thus the higher dimensional gauge invariance affords a protection mechanism. The basic steps of understanding are as follows:

- A 5d gauge field $A_{M}$ can be decomposed as $\left(A_{\mu}, A_{5}\right)$, where $\mu=0,1,2,3$. Can $A_{5}$ be identified with Higgs?

Let us consider a $5 \mathrm{~d}$ QED on $S^{1} / Z_{2}$ as a simple example. It does not work as none of the $A_{5}^{(n)}$ survives as a physical state. Each of them is 'eaten up' by the corresponding $A_{\mu}^{(n)}$ so that the latter becomes massive.

- Start with $\mathrm{SU}(3)$ as a gauge group. Suppose that an orbifold projection breaks it to $\mathrm{SU}(2) \times$ $\mathrm{U}(1)$. This can achieved by a projection matrix $P$, that in the fundamental representation, is $P=\operatorname{diag}(-1,-1,1)$.

Denote the SU(3) generators by $T^{a}$ where $a=1, \ldots, 8$. Now, impose $Z_{2}$ projection such that the Lie-algebra valued $A_{\mu} \equiv A_{\mu}^{a} T_{a}$ and $A_{5} \equiv A_{5}^{a} T_{a}$ fields transform as $P A_{\mu} P^{\dagger}=A_{\mu}$ and $P A_{5} P^{\dagger}=-A_{5}$.

Due to the relative minus sign between the two sets of transformations, while the massless gauge bosons transform in the adjoint of $\mathrm{SU}(2) \times \mathrm{U}(1)$, the massless scalars transform as a complex doublet under $\mathrm{SU}(2) \times \mathrm{U}(1)$. This complex doublet can be identified with the Higgs doublet.

- Of course, the next question is how to generate the electroweak scalar potential $V$ involving this Higgs. This potential is forbidden at tree level due to the shift symmetry of the scalar $A_{5}$ fields. In other words, higher dimensional gauge invariance forbids this potential at tree level.

- The interaction of the Higgs with bulk fermions and gauge bosons will generate an effective scalar potential $V$ at the one loop level. The $\mathrm{SU}(2) \times \mathrm{U}(1)$ symmetry will break to $\mathrm{U}(1)_{\mathrm{em}}$. The one loop Higgs mass will be given by

$$
m_{h}^{2} \sim \frac{g^{4}}{128 \pi^{6}} \frac{1}{R^{2}} \sum V^{\prime \prime}(\alpha),
$$

where $\alpha$ is some dimensionless parameter which arises from the bulk interactions, and the sum is over all KK fields.

- A snapshot of the spectrum is the following:

$M_{W}^{(n)}=(n+\alpha) / R, M_{Z}^{(n)}=(n+2 \alpha) / R, M_{\gamma}^{(n)}=n / R$.

The periodicity property demands that the spectrum will remain invariant under $\alpha \rightarrow \alpha+1$. This restricts $\alpha=[0,1]$. Orbifolding further reduces it to $\alpha=[0,0.5]$. Experimentally, $\alpha$ can be fixed from the $W$ mass. 
Clearly, the above scenario does not work as it leads to $M_{Z}=2 M_{W}$. Still, it provides the basic intuitive framework how to proceed.

Instead of getting into the details of a more phenomenologically allowed scenario [1], we just mention three most difficult obstacles that one faces. The GHU scenarios often lead to (i) too small a top quark mass, (ii) too small a Higgs mass, and (iii) too low a compactification scale! Besides handling these, one has also to worry about generating hierarchical Yukawa interaction starting from gauge interaction in higher dimension which, of course, is universal.

\section{Little Higgs mechanism}

A. Cohen gave a talk and led the discussion, based on the work [2] . Electroweak precision data suggest that the Higgs had better been light. But why is it so? Unlike the masses of the gauge bosons and fermions the Higgs mass is not protected by any symmetry in the standard model (SM). The 'Little Higgs' (LH) models provide such a symmetry conceiving Higgs as a pseudo-Goldstone boson (pGB) whose mass is protected by the approximate nature of the global symmetry. The essential points are the following:

- Any LH model has a number of global symmetries, any one of which alone can keep the Higgs massless. Gauge and Yukawa interactions break the symmetry, generating Higgs mass at one-loop level. No single operator (spurion) can break all the symmetries, it has to be 'collective'.

- Start with a global group $G$ which breaks to $H$ with a decay constant $f$. The origin of this symmetry breaking is irrelevant below the cutoff scale $\Lambda \sim 4 \pi f$. $H$ must contain $\mathrm{SU}(2) \times$ $\mathrm{U}(1)$ as a subgroup so that when a part of $G$ is weakly gauged the unbroken SM group results. The Higgs - doublet under the SM group - is a 'part' of the Goldstone boson multiplet which parametrizes the coset space $G / H$. [For instance, $\mathrm{G}=\mathrm{SU}(5)$ and $\mathrm{H}=\mathrm{SO}(5)$ ]. However, the generators of the gauged part of $G$ do not commute with the generators corresponding to the Higgs, and thus gauge (as well as Yukawa) interactions induce Higgs mass at one-loop level.

- We require $m_{h} \sim f / 4 \pi \sim 100 \mathrm{GeV}$. Hence $f \sim 1 \mathrm{TeV}$. The cutoff is then $\Lambda=4 \pi f \sim 10 \mathrm{TeV}$.

- A clever construction of a LH theory should have the following form of the electroweak sector Higgs potential:

$$
V(h)=-\mu^{2}\left(h^{\dagger} h\right)+\lambda\left(h^{\dagger} h\right)^{2},
$$

where, the bilinear term is suppressed, $\mu^{2} \sim \frac{g^{4}}{16 \pi^{2}} f^{2} \ln \left(\Lambda^{2} / f^{2}\right)$, but, crucially, the quartic interaction should be unsuppressed, $\lambda \sim g^{2}$. This is achievable in the $\mathrm{SU}(5) / \mathrm{SO}(5)$ model.

- Thus the Higgs mass has a log sensitivity to the cutoff at one-loop (but quadratic sensitivity at two-loop) which is sufficient to keep the electroweak scale of $246 \mathrm{GeV}$ natural. The $S$ and $T$ parameters can be kept under control.

- The 'smoking gun' signals will constitute a few weakly coupled particles (gauge bosons, top-like quark and a scalar coupled to the Higgs) below a TeV. 


\section{3. $W_{L}-W_{L}$ scattering}

\section{S.D. Rindani gave a talk and led the discussions.}

In the absence of a light Higgs, $W W$ interactions become strong at TeV scales. If $m_{h}>700 \mathrm{GeV}$, such amplitudes violate perturbative unitarity at high energy and the gauge sector becomes strongly interacting. New interactions can restore unitarity though. Thus, the study of $W W$ scattering can give information of the electroweak symmetry breaking sector and discriminate between models. In general there are large cancellations between the scattering and bremsstrahlung diagrams. Hence extraction of $W W$ scattering contribution from the process $P P \rightarrow W^{+} W^{-} X$ needs considerable effort, especially since the equivalent vector boson approximation results in a gross overestimation. Furthermore, the backgrounds need to be reduced by an appropriate choice of cuts. It is possible to extract information on $W W$ scattering from hadronic experiments by concentrating on the largeinvariant mass region [3].

\section{Mass, mass-shifts, Higgs}

Y.N. Srivastava gave a talk and led the discussions.

The discussion is based on a claim that the masses of heavy particles (comparable to $v=246$ $\mathrm{GeV}$ ), such as $Z$ and $W$ bosons and top quark, depend on the process by which they are measured! According to [4, 5], particles produced singly are predicted to have higher masses than when they are produced in association with other particles (e.g. when they are pair-produced). Based on this, the speaker discussed a new technique to look for the Higgs boson which through its nature as a 'field' induces mass shifts to other particles [6]. The details can be found in the above references.

\section{Top quark spin correlations}

K. Smolek gave a talk and led the discussions.

With the LHC producing millions of top, various precision studies would be possible. In particular, with the top decaying before it hadronizes, its decay products retain infomation about its polarization and, thus, about the production process. For any given decay mode, the normalized differential distribution in $\theta_{f}$, the angle, in the top rest frame, between the top polarization and the direction of motion of the decay product $f$ can be parameterized as

$$
\frac{1}{\Gamma} \frac{d \Gamma}{d \cos \theta_{f}}=\frac{1}{2}\left(1+\alpha_{f} \cos \theta_{f}\right) .
$$

The coefficient $\alpha_{f}$, called the top spin analyzing power, is a constant between -1 and 1 . For the charged lepton, $\alpha_{l^{+}}=+1$ at tree level, while $\alpha_{b}=-0.41$ for the $b$-quark and $\alpha_{v_{l}}=-0.31$ for the $v_{l}$ respectively. In hadronic decay modes, the role of the charged lepton is replaced by the $d$ or $s$ quark.

Since $\alpha_{f}$ is maximal for leptons and since it is easy to distinguish leptons from antileptons, the best way to analyze the $t \bar{t}$ spin correlations is to look for angular correlations in the two charged leptons, when both $t$ and $\bar{t}$ decay leptonically. The decay distribution above could then be folded with the production matrix element squared to obtain the requisite density matrices. Remembering 
that, even for QCD production, the number of $t \bar{t}$ pairs with same helicity for either is not the same as that with opposite, one may define an asymmetry

$$
\mathscr{A}=4\left\langle\left(\hat{p}_{t} \cdot \vec{S}_{t}\right)\left(\hat{p}_{\bar{t}} \cdot \vec{S}_{\bar{t}}\right)\right\rangle=\frac{\sigma\left(t_{\uparrow} \bar{\uparrow}_{\uparrow}\right)+\sigma\left(t_{\downarrow} \bar{t}_{\downarrow}\right)-\sigma\left(t_{\uparrow} \bar{t}_{\downarrow}\right)-\sigma\left(t_{\downarrow} \bar{\uparrow}_{\uparrow}\right)}{\sigma\left(t_{\uparrow} \bar{\tau}_{\uparrow}\right)+\sigma\left(t_{\downarrow} \bar{t}_{\downarrow}\right)+\sigma\left(t_{\uparrow} \bar{\uparrow}_{\downarrow}\right)+\sigma\left(t_{\downarrow} \bar{\uparrow}_{\uparrow}\right)} .
$$

Clearly, if new physics is relevant in top production at the LHC, the measured value of $\mathscr{A}$ would deviate from its SM prediction of 0.319 (when integrated over the entire phase space).

Using the aforementioned density matrix, the double differential distribution for a particular pair of daughters arising from each of $t$ and $\bar{t}$ decay can be parametrized as

$$
\frac{1}{N} \frac{d^{2} N}{d \cos \theta_{f} d \cos \theta_{\bar{f}}}=\frac{1}{4}\left(1-\mathscr{A} \alpha_{f} \alpha_{\bar{f}} \cos \theta_{f} \cos \theta_{\bar{f}}\right),
$$

Using an unbiased statistic of the form $\mathscr{A}=-9\left\langle\cos \theta_{f} \cos \theta_{\bar{f}}\right\rangle$, one may then estimate the resolving power of the LHC for various new physics scenarios. It should be noted here that, for a given scenario (including the SM), the value of $\mathscr{A}$ is not a constant but depends on the phase space restrictions imposed. This can, in principle, be used to one's advantage.

In particular, the above formalism can be used for spin-2 ( $s$-channel) intermediate states in both $g g \rightarrow t \bar{t}$ and $q \bar{q} \rightarrow t \bar{t}$. Concentrating first on the case of large extra dimensions, the spin-2 particles are nothing but the extremely close-spaced Kaluza-Klein tower of gravitons. As is well known, the summation over the tower must be regularized thereby introducing an ambiguity in the expectations. For either case, the expected value for $\mathscr{A}$ would depend on the parameters of the theory. For low values of the ADD scale, $\mathscr{A}$ receives very large negative corrections, reaching downto $\mathscr{A} \sim-0.02$ for $m_{\mathrm{ADD}} \sim 600 \mathrm{GeV}$. For large $m_{\mathrm{ADD}}$, the effect expectedly dies down and $\mathscr{A}$ reverts to its SM value [7].

For the Randall-Sundrum case too, the exchange of the spin-2 gravitons leads to a large negative contribution to $\mathscr{A}$. However, since the KK gravitons are well-separated, one may attempt to increase the sensitivity by measuring $\mathscr{A}$ as a function of the $t \bar{t}$ invariant mass. Within the SM, $\mathscr{A}\left(m_{t \bar{t}}\right)$ decreases montonically with $\mathscr{A}(500 \mathrm{GeV}) \sim 0.42$ and $\mathscr{A}(3000 \mathrm{GeV}) \sim-0.6$. The behaviour in the RS model is more complex though. While, on an average, it continues to fall, around each graviton resonance $\mathscr{A}$ oscillates [7]. The amplitude of oscillations expectedly falls for higher resonances as also with an increasing value of the RS parameter ratio $\kappa / M_{P l}$.

In summary, the measurement of top polarization gives an additional and sensitive probe to new physics process contributing to $t \bar{t}$ production. This is particularly true for (though not restricted to) resonances going into $t \bar{t}$ and is demonstrated here in the context of spin- 2 gravitons.

\section{Single top production at the Tevatron}

V. Simak gave a talk on single top production at DO [8] and led the discussions.

While QCD-mediated pair production is the dominant process for top production at hadronic colliders, electroweak processes also for single top production. The dominant contributions, at the Tevatron, accrue from the $s$-channel process $u+\bar{d} \rightarrow t+\bar{b}$ and the $t$-channel one $u+g \rightarrow t+d+\bar{b}$. 
Summing over the conjugate processes as well, the corresponding NLO cross sections are expected to be $\sigma(p \bar{p} \rightarrow t b X)=0.88 \pm 0.11 \mathrm{pb}$ and $\sigma(p \bar{p} \rightarrow t q b+X)=1.98 \pm 0.25 \mathrm{pb}$ [9, 10].

Such events can be used to study the Wtb coupling, and to directly measure the magnitude of the CKM matrix element $\left|V_{t b}\right|$. This, in turn, can be used to determine the partial decay width $\Gamma(t \rightarrow W b)$ and thus the top lifetime. Furthermore, given the fact that the SM amplitude is an weak one, this mode is also suitable for studying possible beyond the SM (BSM) effects.

The current study is based on $0.9 \mathrm{fb}^{-1}$ of data collected by the D0 detector at $\sqrt{s}=1.96 \mathrm{TeV}$ and with triggers comprising a jet and an $e^{ \pm} / \mu^{ \pm}$. The final state was required to consist of one high transverse momentum $\left(p_{T}\right)$ isolated lepton and missing transverse energy $\left(E_{T}\right)$, together with a $b$-quark jet from the decay of the top quark $(t \rightarrow W b \rightarrow \ell v b)$. The $s$-channel process has an additional $b$ quark, while the $t$-channel one had an additional light quark and a $b$ quark (the last being rarely reconstructed as it is produced in the forward direction with a low $p_{T}$ ). Backgrounds emanate from (i) $W+$ jets; (ii) $t \bar{t}$ decaying into the lepton+jets and dilepton final states, with a jet/lepton not being reconstructed; (iii) multijet production, where a jet is misreconstructed as an electron, or a heavy-flavor quark decays to a muon passing the isolation criteria.

The signal was modeled with a combination of the SINGLETOP NLO Monte Carlo event generator [11] and PYTHIA. For the $t b$ search, SM $t q b$ was treated as part of the background, and vice versa. For the $t b+t q b$ search, the SM ratio between the $t b$ and $t q b$ cross sections was assumed. The $t \bar{t}$ and $W+$ jets backgrounds were simulated using the ALPGEN leading-order MC event generator [12] and PYTHIA and a parton-jet matching algorithm [13] used to eliminate double-counting. The multijet background is modeled using data containing nonisolated leptons. The $W+$ jets background, combined with the multijet background, is normalized to the lepton+jets dataset separately for each analysis channel before $b$-jet tagging.

Jets were reconstructed using the cone algorithm with radius $\mathscr{R}=\sqrt{(\Delta y)^{2}+(\Delta \phi)^{2}}=0.5$ (where $y$ is rapidity and $\phi$ is azimuthal angle) to cluster energy deposits in the calorimeter and were required to have $p_{T}>15 \mathrm{GeV}$ and $|\eta|<3.4$. In addition, the leading jet has $p_{T}>(20) 25 \mathrm{GeV}$ and $|\eta|<2.5$ while the second leading jet has $p_{T}>20 \mathrm{GeV}$. Events were required to have $15<$ $Z_{T}<200 \mathrm{GeV}$ and exactly one isolated electron with $p_{T}>15 \mathrm{GeV}$ and $|\eta|<1.1$ or one isolated muon with $p_{T}>18 \mathrm{GeV}$ and $|\eta|<2.0$. Requiring that the direction of the $E_{T}$ is not aligned or anti-aligned in azimuth with the lepton or a jet rejects misreconstructed events. To enhance the signal content, one or two of the jets are required to be identified as originating from long-lived $b$ hadrons using a combination of secondary vertex information and neural networks.

Since single top events would constitute only a small fraction of the selected event samples, and since the background uncertainty is large, a counting experiment does not suffice. Instead, multivariate discriminants that separate the signal from background are considered and decision trees [14] used. Forming a binned likelihood as a product over all bins and channels of the decision tree discriminant, separately for the $t b+t q b, t q b$, and $t b$ analyses, a Bayesian approach is adopted for measuring the production cross section. Assuming a Poisson distribution for the observed counts and flat nonnegative prior probabilities for the signal cross sections, and accounting for systematic uncertainties and their correlations by integrating over the signal acceptances, background yields, and integrated luminosity with Gaussian priors for each, the final posterior probability density is computed as a function of the production cross section.

A $3.4 \sigma$ excess of events over the background was found in the high discriminant output region. 
Interpreting it as evidence for single top quark production, the resultant cross sections are $\sigma(p \bar{p} \rightarrow$ $t b+X, t q b+X)=4.9 \pm 1.4 \mathrm{pb}, \sigma(p \bar{p} \rightarrow t q b+X)=4.2_{-1.4}^{+1.8} \mathrm{pb}$, and $\sigma(p \bar{p} \rightarrow t b+X)=1.0 \pm$ $0.9 \mathrm{pb}$, with uncertainties including both statistical and systematic components (for the tb+tqb sample, statistics contribute $1.2 \mathrm{pb}$ to the uncertainty).

While $\sigma_{\text {meas. }}>\sigma_{\mathrm{SM}}$, they are consistent within the (presently large) errors. This, however, can be used to derive a first direct measurement of the strength of the coupling $\left|V_{t b} f_{1}^{L}\right|$ in the Wtb vertex, where $f_{1}^{L}$ is an arbitrary left-handed form factor. Assuming $\left|V_{t d}\right|^{2}+\left|V_{t s}\right|^{2} \ll\left|V_{t b}\right|^{2}$ and a pure $V-A$ and CP-conserving $W t b$ interaction, this yields $\left|V_{t b} f_{1}^{L}\right|=1.3 \pm 0.2$. Assuming in addition that $f_{1}^{L}=1$ and using a flat prior for $\left|V_{t b}\right|^{2}$ from 0 to 1 , one obtains $0.68<\left|V_{t b}\right| \leq 1$ at $95 \%$ C.L. These measurements make no assumptions about the number of quark families or CKM matrix unitarity.

Finally, it should be noted that an accurate measurement of this channel is of great importance as it constitutes an important background to Higgs search.

\section{References}

[1] G. Cacciapaglia, C. Csaki and S. C. Park, JHEP 0603 (2006) 099 [arXiv:hep-ph/0510366]; G. Panico, M. Serone and A. Wulzer, Nucl. Phys. B 739 (2006) 186 [arXiv:hep-ph/0510373].

[2] N. Arkani-Hamed, A. G. Cohen, E. Katz and A. E. Nelson, JHEP 0207 (2002) 034 [arXiv:hep-ph/0206021]; N. Arkani-Hamed, A. G. Cohen, E. Katz, A. E. Nelson, T. Gregoire and J. G. Wacker, JHEP 0208 (2002) 021 [arXiv:hep-ph/0206020]; N. Arkani-Hamed, A. G. Cohen, T. Gregoire and J. G. Wacker, JHEP 0208 (2002) 020 [arXiv:hep-ph/0202089]; N. Arkani-Hamed, A. G. Cohen and H. Georgi, Phys. Lett. B 513 (2001) 232 [arXiv:hep-ph/0105239].

[3] See, S.D. Rindani, in this volume, for details.

[4] S. Reucroft and Y. N. Srivastava, Eur. Phys. J. C 48 (2006) 781 [arXiv:hep-ph/0509151].

[5] S. Reucroft, Y. N. Srivastava, J. Swain and A. Widom, arXiv:hep-ph/0511233.

[6] S. Reucroft, Y. Srivastava, J. Swain and A. Widom, J. Phys. Conf. Ser. 60 (2007) 187 [arXiv:hep-ph/0611066].

[7] M. Arai, N. Okada, K. Smolek and V. Simak, Phys. Rev. D 75(2007) 095008 [arXiv:hep-ph/0701155]; also, Phys. Rev. D 70 (2004) 115015 [arXiv:hep-ph/0409273].

[8] V. M. Abazov et al. [D0 Collaboration], Phys. Rev. Lett. 98 (2007) 181802 [arXiv:hep-ex/0612052].

[9] Z. Sullivan, Phys. Rev. D 70 (2004) 114012.

[10] N. Kidonakis, Phys. Rev. D 74 (2006) 114012. For $m_{t}=175 \mathrm{GeV}$, $\sigma(p \bar{p} \rightarrow t b+X, t q b+X)=3.21 \pm 0.21 \mathrm{pb}$.

[11] E.E. Boos et al., Phys. Atom. Nucl. 69 (2006) 1317.

[12] M.L. Mangano et al., J. High Energy Phys. 0307 (2003) 001. We used ALPGEN version 2.05.

[13] S. Höche et al., in Proceedings of the Workshop on the Implications of HERA for LHC Physics, edited by A. De Roerck and H. Jung (CERN, Geneva, 2005), p. 288.

[14] L. Breiman et al., Classification and Regression Trees (Wadsworth, Stamford, 1984); D. Bowser-Chao and D.L. Dzialo, Phys. Rev. D 47 (1993) 1900;

Y. Freund and R.E. Schapire, in Proc. XIII ${ }^{\text {th }}$ Inter. Conf. on Machine Learning, ed. L. Saitta (Morgan Kaufmann, San Fransisco, 1996), p. 148; B.P. Roe et al., Nucl. Instrum. Methods A 543 (2005) 577; H.-J. Yang, B.P. Roe, and J. Zhu, Nucl. Instrum. Methods A 555 (2005) 370. 